\title{
Detección de gases traza por técnica fotoacústica basada en un esquema de excitación chirp lineal
}

\author{
Trace gases detection by photoacoustic technique based on a lineal chirp excitation scheme \\ Carlos G. Carreño Romano*, Germán A. Pérez Fogwill*, G. D. Santiago*, M. G. González*†1 \\ * Universidad de Buenos Aires, Facultad de Ingeniería, \\ Grupo de Láser, Óptica de Materiales y Aplicaciones Electromagnéticas (GLOMAE) \\ Paseo Colón 850, C1063ACV, Buenos Aires, Argentina \\ ${ }^{\dagger}$ Consejo Nacional de Investigaciones Científicas y Técnicas, (CONICET) \\ Godoy Cruz 2290, C1425FQB, Buenos Aires, Argentina \\ ${ }^{1}$ mggonza@fi.uba.ar \\ Recibido: 16/04/18; Aceptado: 17/05/18
}

\begin{abstract}
We present a new photoacoustic gas-trace measurement setup, based on a chirped optical chopper. This method combines features of the resonant and pulsed techniques. To show the advantages of this setup, we carried out a comparison with the resonant method in samples of $\mathrm{NO}_{2}$ contained in a one-dimensional acoustic resonator. The results show the chirped technique allows carrying out short-term acquisitions with good signal-to-noise ratio.
\end{abstract}

Keywords: Photoacoustic spectroscopy; trace gases; chirp.

Resumen- En este trabajo se presenta un nuevo esquema de medición para espectroscopía fotoacústica de gases traza basado en un esquema de excitación up - chirp. Esta forma combina las ventajas de los esquemas resonante y pulsado. Para mostrar el potencial de esta técnica se la comparó con un esquema de excitación resonante sobre la medición de muestras de dióxido de nitrógeno en aire contenidas en una resonador acústico de aluminio. Los resultados mostraron que la técnica chirp permite realizar mediciones de corta duración con muy buena relación señal a ruido.

Palabras clave: Espectroscopía fotoacústica; gases traza; chirp.

\section{INTRODUCCIÓN}

La detección de gases traza es un tema de primordial interés debido a la importancia que reviste en distintas áreas, muchas de ellas directamente relacionadas con el cuidado de la vida en nuestro planeta. Como ejemplo basta nombrar los estudios ambientales referidos al cambio climático, la destrucción de ozono estratosférico y formación de smog fotoquímico, las técnicas de diagnóstico en medicina, donde es importante el óxido de nitrógeno generado en algunos tejidos mamíferos como mediador de respuestas fisiológicas y patológicas y, en agronomía, la medición de emanaciones de hormonas gaseosas, como el etileno, bajo condiciones de stress de plantas y de difusión de gases en las mismas durante períodos de anegamiento [1].

En lo que se refiere a estudios atmosféricos, cantidades relativamente pequeñas de ciertos gases tienen gran impacto sobre el medio ambiente y el cambio climático. El estudio de la atmósfera, su química y los procesos de transporte e intercambio requiere un monitoreo permanente con técnicas adecuadas para cada caso, ya que hay una gran variedad de contaminantes cuyas concentraciones van desde algunos por cientos $\left(10^{-2}\right)$ hasta partes por trillón $\left(10^{-12}\right)$. Idealmente estas técnicas de detección deberían cumplir con los requisitos de [1] i) alta sensibilidad para medir muy bajas concentraciones; ii) alta selectividad para diferenciar distintos componentes en mezclas; iii) buena resolución temporal para estudiar fluctuaciones, y iv) portabilidad, para realizar mediciones in situ. La importancia de cada uno de estos ítems varía según la situación.

Se han desarrollado diferentes esquemas de monitoreo novedosos que tienen su origen en el progreso de la investigación en física y química, en especial en materiales y óptica, así como de la electrónica y computación. En el tema ambiental, éstos fundamentalmente pueden distinguirse en el proceso de toma de muestras y en el método analítico para determinación de especies moleculares y medición de concentraciones.

Respecto de la toma de muestras, la técnica puede ser: por extracción, monitoreo in situ o monitoreo remoto. En el primer caso, la muestra se recoge en recipientes y es llevada al laboratorio. Los métodos que se pueden implementar allí son en general más sensibles que los aplicables en campo pero no permiten el estudio en tiempo real, como el segundo caso. En el tercer caso, el equipo, basado en sistemas ópticos, es instalado lejos del lugar bajo estudio por lo que evita la interacción del aparato con la muestra durante el monitoreo pero por otro lado depende fuertemente de las condiciones de retrodispersión atmosférica.

En referencia a los métodos analíticos para detección de gases en general se encuentran las técnicas espectroscópicas que resultan muy versátiles y están principalmente basadas en la utilización de láseres ya que son fuentes de luz intensas sintonizables. En este caso, la medición de concentración se realiza a partir de la interacción radiación-materia que puede consistir en un proceso de dispersión o absorción de la luz. Uno de los fenómenos que más ventajas ofrece para la identificación de especies moleculares y determinación de su concentración es la absorción de radiación. Ésta se produce en especial en los rangos del IR medio $(2-25 \mu \mathrm{m})$ y del visible a UV (700-250 nm). Diferentes sistemas de medición están fundamentalmente basados en un esquema simple y en la aplicación de la ley de Beer-Lambert [2].

Una técnica muy sensible y selectiva, que permite de- 
tección de gases traza in situ, está basada en efectos térmicos provocados por absorción de radiación. Generalmente, de acuerdo con los detectores utilizados para medir esto, existen dos esquemas [1]. El primero de ellos corresponde a espectroscopía fotoacústica (EFA). En este caso el detector puede ser un micrófono [2] o un diapasón de cuarzo [3] los cuales son utilizados para captar la onda acústica que se genera cuando parte de la energía absorbida se convierte en calor. Por otro lado, está la espectroscopía fototérmica (EFT) donde, por ejemplo, se utiliza un determinado sistema de detección que permite captar el cambio de índice de refracción producido por el calentamiento de la muestra [4].

Tanto en EFA como en EFT, la muestra gaseosa bajo estudio se encuentra habitualmente confinada dentro de un recinto que actúa como un resonador acústico y es excitada por un láser a través de una o más ventanas. La excitación puede ser efectuada de dos maneras [5]. En la primera, el haz láser es modulado (usualmente con un obturador mecánico) a una frecuencia próxima a la de alguna de las resonancias acústicas del recinto. Esta forma es denominada excitación resonante y conduce naturalmente a la detección sincrónica de la señal acústica por medio de un amplificador de tipo $l o c k-i n$, por la excelente relación señal a ruido asequible. Cambios en la temperatura o composición de la muestra ocasionan un corrimiento de las frecuencias de resonancia, por lo cual resulta crítico el enganche de la frecuencia de modulación del haz láser con las mismas. La otra forma de excitación, denominada pulsada, consiste en registrar la señal del micrófono resuelta en tiempo luego de la excitación de la muestra con pulsos de luz de alta intensidad y corta duración. En este caso, el análisis de la señal se puede realizar en el dominio de las frecuencias a través de la transformada Fourier de la señal temporal. Así, se evita la sincronización de la frecuencia de modulación con las frecuencias de resonancia de la celda ya que con un solo pulso láser se obtienen todas las frecuencias de oscilación de la cavidad acústica. De esta manera los corrimientos de frecuencia debido a cambios de temperatura o composición dejan de ser importantes. Sin embargo, todo lo anterior es a expensas de una menor sensibilidad debido a una menor relación señal a ruido [6]. Esto último usualmente implica la utilización de pulsos de luz de gran energía, lo que dificulta la implementación de esquemas FA compactos y portables.

En la EFA de gases traza existe un fenómeno que, de no ser tenido en cuenta, puede deteriorar fuertemente la sensibilidad del sistema. Éste es el proceso de adsorcióndesorción (sorción), el cual se encuentra presente en el caso de moléculas polares como el agua, el amoníaco o el dióxido de nitrógeno. La sorción puede producir importantes variaciones en las muestras durante su análisis. Este fenómeno ha sido estudiado por varios autores, por ejemplo [7] y [8]. Los mismos han recurrido a la utilización de flujo del gas, evaluando la velocidad óptima para minimizar la adsorción y a la vez los ruidos generados por el movimiento del gas. Sin embargo, existen muchos casos donde la muestra se encuentra en cantidad reducida o el dispositivo no puede trasladarse para realizar la medición in situ y, entonces, no puede aplicarse esta solución sino que la medición debe realizarse de forma estanca. Para estos casos, trabajos como el de la referencia [9], se usan modelos que describen

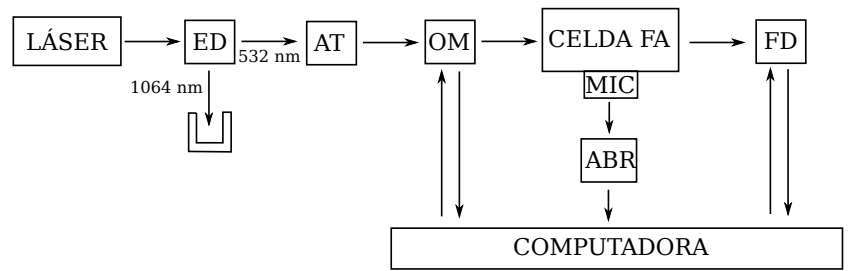

Fig. 1. Esquema experimental. ED: espejo dicroico. AT: atenuador óptico. OM: obturador mecánica. MIC: micrófono. ABR: amplficador de bajo ruido. FD: fotodiodo.

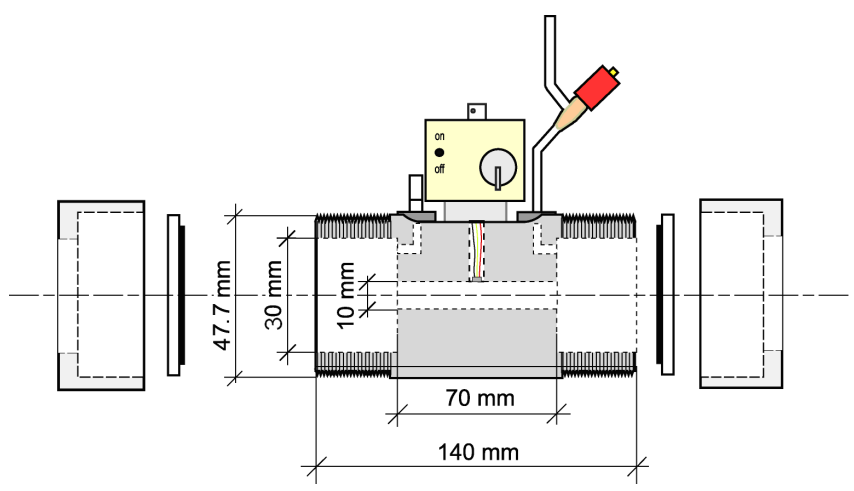

Fig. 2. Esquema de la celda FA usada para las mediciones. Este resonador posee una entrada de gases ubicada en un nodo de presión para evitar distorsiones acústicas. La tapas son desmontables para cambiar las ventanas de entrada de acuerdo a la longitud de onda del láser utilizado para excitar la muestra. En la caja metálica, ubicada al lado de la entrada de gases, se encuentra el circuito que polariza al micrófono electreto.

el proceso de sorción, de manera de poder extrapolar los resultados de la medición.

En este trabajo presentamos un esquema FA que combina las ventajas de las excitaciones resonante y pulsada. Este se basa en que un haz láser es modulado en amplitud pero su frecuencia es incrementada linealmente durante una ventana de tiempo. Esta técnica es conocida como up - chirp. Los valores de duración y frecuencias iniciales y finales del barrido son escogidos de manera de minimizar los problemas antes mencionados: relación señal a ruido, cambio de la frecuencia resonancia y sorción de la muestra. Para comprobar esto implementamos una sistema FA para medición de trazas de $\mathrm{NO}_{2}$ en aire que utiliza un obturador mecánico que permite la medición con un esquema resonante y con el esquema $u p-c h i r p$.

\section{Sistema Fotoacústico}

En la Fig. 1 se muestra el esquema experimental implementado. Cómo fuente de luz se utilizó un láser Nd:YAG continuo con generador de segunda armónica. Para remover las componentes de luz no deseadas pertenencientes a longitud de onda de $1064 \mathrm{~nm}$ se usó un espejo dicroico y la potencia media de luz fue ajustada con un atenuador óptico. La modulación en amplitud y en frecuencia del láser se consiguió con un obturador mecánico controlado por computadora realizado en nuestro laboratorio. El haz modulado fue dirigido a una celda FA conteniendo la muestra absorbente. Un micrófono electreto (Knowles EK3132), junto con un amplificador de bajo ruido (Tektronix AM-502), fueron los encargados de captar la señal acústica generada. Para relevar la intensidad y variación temporal 
del haz láser se usó un fotodiodo calibrado (BPW-34). Las señales provenientes del micrófono y del fotodiodo fueron digitalizadas por una placa de sonido estándard con entrada de línea y frecuencia de muestreo de $44.1 \mathrm{kHz}$.

Las muestras gaseosas se armaron con un línea de vacío que permitió la realización de mezclas de $\mathrm{NO}_{2}$ con aire seco. Las concentraciones del gas absorbente usadas estuvieron en el rango entre 100 y 1000 ppm. Se escogió $\mathrm{NO}_{2}$ ya que su cuantifición presenta gran interés debido a que es un agente contaminante urbano producido principalmente por motores que genera varios problemas de salud respiratorios así como también lluvia ácida [1]. Además, como se detalló en la introducción, el $\mathrm{NO}_{2}$ es una molécula polar que tiene una importante sorción con el material de la celda FA usada para las mediciones.

Como resonador FA se recurrió al uso de una celda unidimensional con filtros de cuarto de onda en sus extremos [10], [11]. En la Fig. 2 se muestra un esquema de la misma que fue construida por el Centro de Investigaciones en Láseres y Aplicaciones de CITEDEF. El material usado para su implementación fue aluminio para el cuerpo y cuarzo para las ventanas que permiten la entrada del haz láser al resonador. La palabra unidimensional denota que el diámetro del resonador es mucho menor que la longitud de onda escogida para que resuene. En cuanto a los filtros, son dos cavidades amortiguadoras o buffers contiguas al resonador que se utilizan con los siguientes propósitos [6]: a) la alta impedancia acústica de las ventanas es transformada a un valor más bajo, permitiendo que el resonador se asemeje a uno abierto; b) la integral de solapamiento de un modo longitudinal es distinta de cero; c) los efectos acústicos de la entrada y salida del gas, localizados en la transición entre el resonador y los buffers, son minimizados;y d) la señal inducida por el calentamiento de las ventanas que alcanza el micrófono es fuertemente reducida.

El diseño de este tipo de celdas es realizado a partir del modo acústico escogido para que resuene. Luego, para cierta temperatura y gas se calcula la longitud de onda. Entonces el largo del resonador y los buffers quedan definidos. Por último, la relación entre los diámetros se escoge para producir la deseada desadaptación entre un medio y otro, consiguiendo, de esta manera, la mayor reflexión posible por parte de la señal proveniente de las ventanas. Para $\mathrm{NO}_{2}$ en aire seco a temperatura ambiente la primera frecuencia de resonancia de la celda se encuentra aproximadamente en $1980 \mathrm{~Hz}$.

\section{UP-CHIRP}

La variación de la intensidad de luz del haz láser generada por el obturador mecánico se puede expresar como:

$$
I(t)=S C(\phi(t))
$$

donde $\phi$ es la función que representa como cambia la fase de la señal y $S C$ es la función onda cuadrada. De esta manera, la frecuencia instantánea es:

$$
f(t)=\frac{1}{2 \pi} \frac{d \phi(t)}{d t}
$$

Basado en las ecuaciones anteriores, la variación de la frecuencia en función del tiempo se puede expresar como:

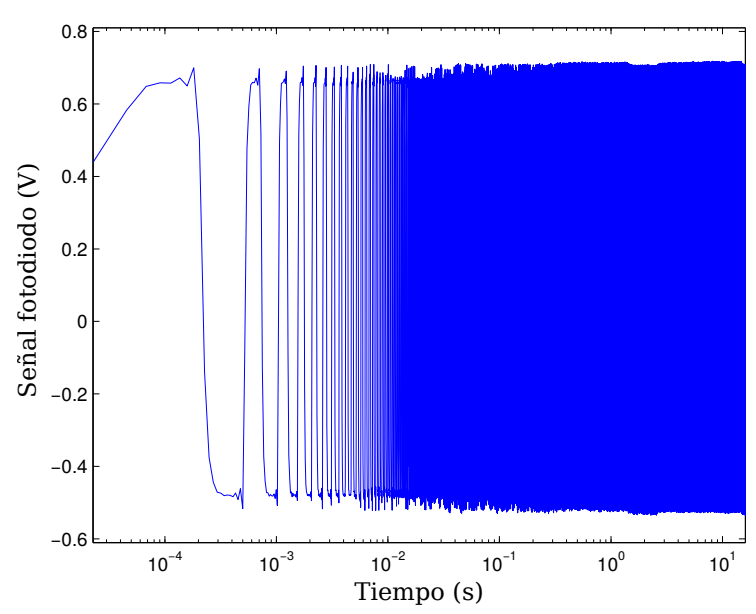

Fig. 3. Señal medida por el fotodiodo para un barrido en frecuencia con parámetros $f_{0}=1920 \mathrm{~Hz}, f_{1}=2030 \mathrm{~Hz}, T=15 \mathrm{~s}$.

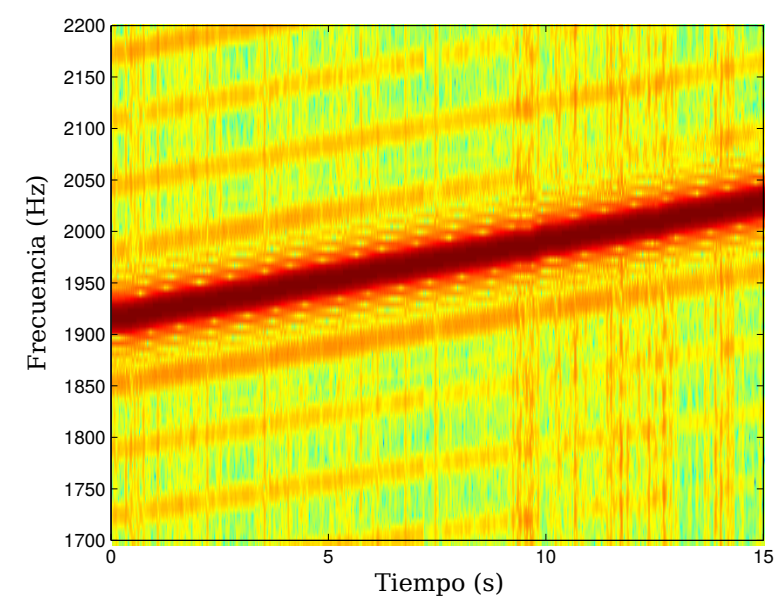

Fig. 4. Espectrograma de señal medida por el fotodiodo para un barrido en frecuencia con parámetros $f_{0}=1920 \mathrm{~Hz}, f_{1}=2030 \mathrm{~Hz}, T=15 \mathrm{~s}$. La intensidad de la trama es proporcional al contenido de energía de la señal en la frecuencia y el tiempo indicados.

$$
c(t)=\frac{d f(t)}{d t}
$$

En el caso de chirp lineal la función $f(t)$ varía linealmente con el tiempo,

$$
f(t)=f_{0}+k t=f_{0}+\left(\frac{f_{1}-f_{0}}{T}\right) t
$$

donde $k$ es la tasa de cambio de la frecuencia o chirpyness, $f_{0}$ y $f_{1}$ las frecuencias inicial y final del barrido, respectivamente, y $T$ el tiempo que se toma para barrer desde $f_{0}$ hasta $f_{1}$. Por lo tanto, para un chirp lineal, $\phi(t)$ toma la siguiente forma:

$$
\phi(t)=\phi_{0}+2 \pi\left(f_{0} t+\frac{k}{2} t^{2}\right)
$$

donde $\phi_{0}$ es la fase inicial.

Los ensayos con el obturador mecánico usado en este trabajo mostraron que, debido al momento de inercia del mismo y al torque del motor, para obtener un barrido lineal 


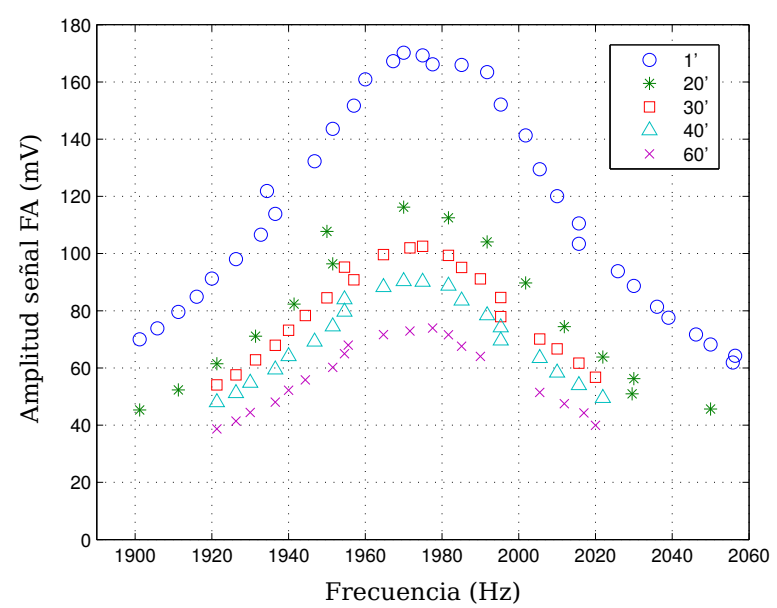

Fig. 5. Amplitud de la señal FA en función de la frecuencia para distintos tiempos tomando como inicio el momento en que se cargó la celda con lcon $600 \mathrm{ppm}$ de $\mathrm{NO}_{2}$ en aire.

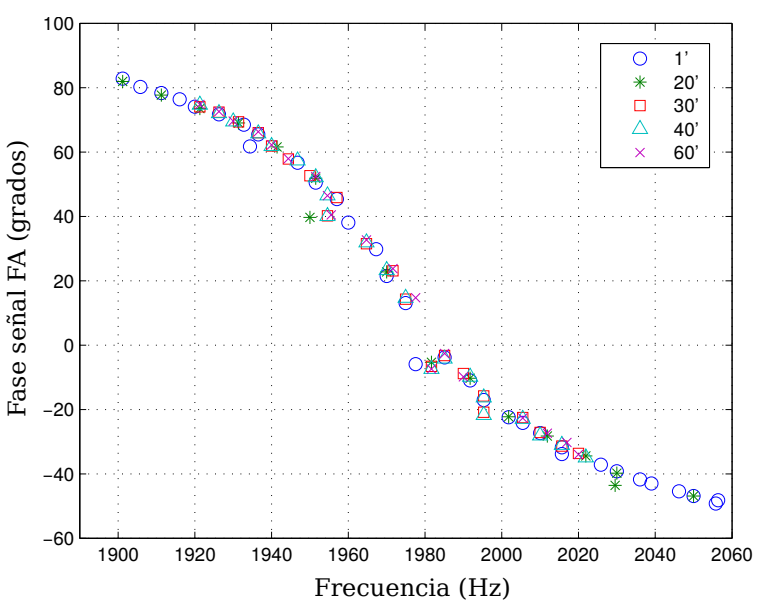

Fig. 6. Fase de la señal FA en función de la frecuencia para distintos tiempos tomando como inicio el momento en que se cargó la celda con 600 ppm de $\mathrm{NO}_{2}$ en aire.

repetitivo de unos $100 \mathrm{~Hz}$ es necesario efectuarlo en un lapso de unos $15 \mathrm{~s}$. Así se obtiene una tasa de unos $7 \mathrm{~Hz} / \mathrm{s}$ Éste resultó el menor tiempo asequible para el obturador. El cambio de frecuencia relativamente lento permite asegurar que la señal acústica dentro del resonador alcanza un virtual régimen estacionario. En efecto, sabiendo que la frecuencia de resonancia es $1980 \mathrm{~Hz}$ y que el factor de calidad de la resonancia es $Q \approx 20$, para un sistema de segundo orden se obtiene un tiempo de establecimiento de unos $10 \mathrm{~ms}$. En este lapso la frecuencia del modulador varía aproximadamente $0.07 \mathrm{~Hz}$, valor despreciable a los efectos prácticos.

En la Fig. 3 se puede apreciar la variación de la intensidad del haz láser medida por el fotodiodo cuando se realiza el barrido en frecuencia con $f_{0}=1920 \mathrm{~Hz}, f_{1}=2030 \mathrm{~Hz}$ y $T=15 \mathrm{~s}$. El redondeo que se ve en los flancos de la señal son causados por la limitación de ancho de banda del sistema de detección. Asimismo, en la Fig. 4, se presenta el espectrograma de los mismos datos donde se hace evidente la variación lineal de la frecuencia con el tiempo.

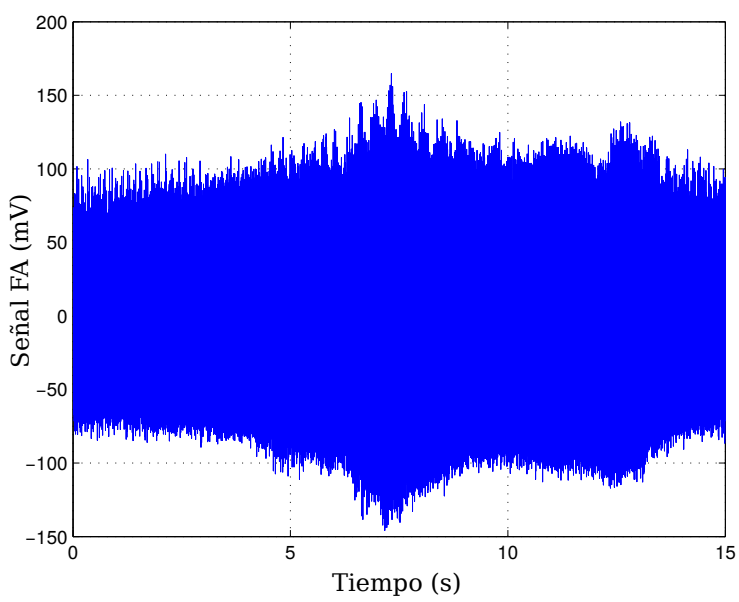

Fig. 7. Señal FA captada por el micrófono bajo el esquema de excitación $u p-$ chirp lineal con parámetros $f_{0}=1920 \mathrm{~Hz}, f_{1}=2030 \mathrm{~Hz}, T=15 \mathrm{~s}$ con la celda conteniendo $600 \mathrm{ppm}$ de $\mathrm{NO}_{2}$ en aire.

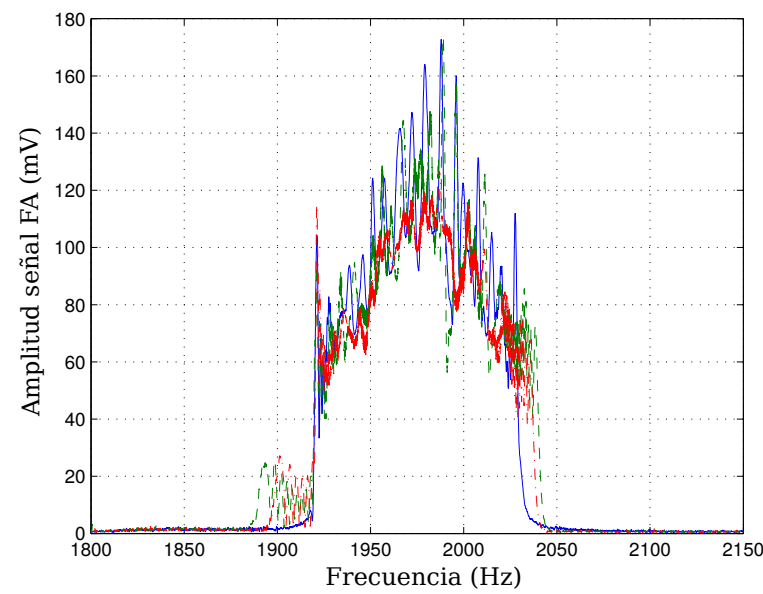

Fig. 8. Amplitud de los espectros de varias señales FA captadas por el micrófono bajo el esquema de excitación $u p$ - chirp lineal con parámetros $f_{0}=1920 \mathrm{~Hz}, f_{1}=2030 \mathrm{~Hz}, T=15 \mathrm{~s}$ con la celda conteniendo $600 \mathrm{ppm}$ de $\mathrm{NO}_{2}$ en aire.

\section{RESUltados}

Para la medición con el esquema de excitación resonante se realizó un barrido en frecuencia en pasos de 5 $\mathrm{Hz}$, alrededor de la frecuencia de resonancia, relevando el módulo y fase de la señal captada por el micrófono. Para el procesamiento de los datos se implementó una detección sincrónica usando el método descripto en [12] que utiliza una placa de sonido para adquirir dos señales: la proveniente del micrófono, previamente amplificada, y la de referencia. A partir de esta última se obtienen dos señales cuasiortogonales que permiten obtener el módulo y fase de la señal FA. Como señal de referencia se tomó la señal de control del obturador mecánico. Se hicieron varios barridos a distintos tiempos para relevar el fenómeno de sorción que presenta el $\mathrm{NO}_{2}$. Se escogió como tiempo inicial el momento donde se cargó la celda con la muestra. En las Figs. 5 y 6 se presentan los resultados obtenidos para $600 \mathrm{ppm}$ de $\mathrm{NO}_{2}$ en aire de donde se desea remarcar que i) a partir de los gráficos de fase se determinó que la frecuencia de 
resonancia medida se encontró en el entorno de $1970 \mathrm{~Hz}$; ii) cada barrido demoró entre 8 y 10 minutos; iii) entre cada barrido se observa una disminución notable de la amplitud debido al fenómeno de sorción, incluso una hora después de haber cargado la celda; y iv) en el primer barrido se puede apreciar una varición alrededor de la resonancia producto de la fuerte sorción inicial.

Para el esquema de excitación up - chirp se realizó un barrido lineal en frecuencia usando los valores $f_{0}=1920$ $\mathrm{Hz}, f_{1}=2030 \mathrm{~Hz}, T=15 \mathrm{~s}$. En la Fig. 7 se presenta la primera medición captada por el micrófono un minuto luego de cargar la celda con una muestra con la misma concentración de $\mathrm{NO}_{2}$ usada en el esquema resonante. Se puede ver un aumento notorio de la amplitud entorno a los 7.5 segundos que, observando el espectrograma de la Fig. 4 se corresponde con una frecuencia de $1970 \mathrm{~Hz}$. Se realizaron varias medidas cada 10 segundos. En la Fig. 8 se muestran sus transformadas de Fourier donde se observó que las señales son distintas de cero solo para la frecuencias donde se realiza el barrido. Para medir la concentración de $\mathrm{NO}_{2}$ se utilizó el siguiente estimador:

$$
V_{F A}=\frac{1}{f_{2}-f_{1}} \int_{f_{1}}^{f_{2}} S_{m e d}(f) \quad d f
$$

donde $S_{\text {med }}(f)$ es la transformada de Fourier de la señal FA medida. Aplicando este estimador sobre las señales medidas se determinó un valor promedio de $59 \pm 0.2 \mathrm{mV}$. En este caso no se observaron grandes variaciones de amplitud entre las señales medidas debido a lo poco que duró todo el proceso de medición.

También se realizaron varias mediciones con la celda conteniendo únicamente aire. En este caso se obtuvo un valor promedio de $11 \pm 4 \mu \mathrm{V}$, lo que denota que la relación señal a ruido es muy buena.

\section{CONCLUSiOnes}

En este trabajo se presentó una nueva técnica de medición para EFA de gases traza basada en el esquema de excitación up-chirp. Esta forma combina las ventajas de los esquemas resonante y pulsado, o sea, mediciones de corta duración con muy buena relación señal a ruido. Para mostrar el potencial de la técnica se lo comparó con un esquema de excitación resonante que solo se diferencia en la señal de control enviada al obturador mecánico que modula la amplitud de la fuente de luz.

Para mostrar la ventajas del sistema chirp se midieron las señales FA obtenidas de la irradiación de dióxido de nitrógeno en aire seco contenido en un resonador acústico. Los resultados mostraron que la técnica chirp permite realizar mediciones rápidas de forma tal de minimizar los efectos de corrimientos de la frecuencia de resonancia por cambios de temperatura y las variaciones en amplitud de la señal FA medida debido a la sorción de la muestra por la paredes del resonador. Esto último permite evitar la necesidad de complejos sistemas de circulación de gases. Además, en caso de necesitar sistemas para EFA compactos y portátiles, se puede lograr reemplazando la fuente láser de estado sólido y el obturador mecánico por diodos emisores de luz (LEDs) [13].

\section{AGRADECIMIENTOS}

Este trabajo fue apoyado por los subsidios de la Universidad de Buenos Aires (UBACyT 20020160100052BA) y de la ANPCyT (PICT 2016-2204). También queremos agradecer al Centro de Investigaciones en Láseres y Aplicaciones de CITEDEF por habernos prestado la celda fotacústica utilizada en las experiencias.

\section{REFERENCIAS}

[1] A. Peuriot, G. Santiago, and V. Slezak, Modelado, métodos de adquisición y procesamiento en la espectroscopía fotoacústica en gases, ser. Láseres: Desarrollos y Aplicaciones. Tomo 2., L. Azcarate, P. Diodati, E. Quel, and V. Slezak, Eds. Buenos Aires, Argentina: UNSAMedita, 2007.

[2] M. Sigrist, Air monitoring by laser photoacoustic spectroscopy, ser. Air Monitoring by Spectroscopic Techniques. New York, USA: John Wiley, 1994.

[3] A. Kosterev and F. Tittel, "Ammonia detection by use of quartzenhanced photoacoustic spectroscopy with a near-ir telecommunication diode laser," Appl. Opt., vol. 43, pp. 6213-6217, 2004.

[4] S. Bialkowski, Photothermal Spectroscopy Methods for Chemical Analysis. New York, USA: John Wiley, 1996.

[5] A. Miklos, P. Hess, and Z. Bozoki, "Application of acoustic resonators in photoacoustic trace gas analysis and metrology," Rev. Sci. Instrum., vol. 72, pp. 1937-1955, 2001.

[6] M. G. Gonzalez, "Análisis y desarrollo de nuevas técnicas de detección optoacústicas," Ph.D. dissertation, Univ. de Buenos Aires, Buenos Aires, Argentina, Mar. 2008.

[7] A. Schmohl, A. Miklos, and P. Hess, "Effects of adsorption-desorption processes in the response time and accuracy of photoacoustic detection of ammonia," Appl. Opt., vol. 40, pp. 2571-2578, 2001.

[8] J. Henningsen and N. Melander, "Sensitive measurement of adsorption dynamics with nonresonant gas phase photoacoustics," Appl. Opt., vol. 36, pp. 7037-7045, 1997.

[9] A. Peuriot, G. Santiago, V. Slezak, and M. G. Gonzalez, "Study of a spherical resonator applied to photoacoustic spectroscopy," Anales AFA, vol. 19, pp. 59-62, 2007.

[10] P. Repond and M. Sigrist, "Photoacoustic spectroscopy on trace gases with continuously tunable co2 laser," Appl. Opt., vol. 35, pp. 4065 4085, 1996.

[11] F. Bijnen, J. Reuss, and F.Harren, "Geometrical optimization of a longitudinal resonant photoacoustic cell for sensitive and fast trace gas detection," Rev. Sci. Instrum., vol. 67, pp. 2914-2923, 1996.

[12] M. G. Gonzalez, G. Santiago, V. Slezak, and A. Peuriot, "Simple synchronic detection at audio frequencies through a pc sound card," Rev. Sci. Instrum., vol. 78, p. 055108, 2007.

[13] R. Bernhardt, G. Santiago, V. Slezak, A. Peuriot, and M. G. Gonzalez, "Differential, led-excited, resonant no 2 photoacoustic system," Sens. Actuator B-Chem., vol. 150, pp. 513-516, 2010. 